\title{
Contributing Ad Hoc Reviewers 2018
}

Rehabilitation Psychology publishes articles that capture the breadth of science and practice in rehabilitation psychology. To achieve this mission, we rely on a diverse group of individuals who serve as ad hoc reviewers. We are grateful to the following individuals for their generous commitment of time and effort for the 2018 volume of Rehabilitation Psychology. If you would like to join this group of individuals by serving as an ad hoc reviewer, please contact the editor at ehde@uw.edu.

Herb Ames

Derek Ryan Anderson

Anne Arewasikporn

Trey William Armstrong

Kaytlin Alysse Bailey

Anna Banik

Martha E. Banks

Sandra Bassett

Harald Baumeister

Lisa Betthauser

John Parle Blake

Arthur Blaser

Patrick Brice

Susanne M. Bruyere

Alison Buchholz"

Julius Burkauskas

\section{Yue Cao}

Jane E. M. Carter

Jacob Yuichung Chan

Reshawna Chapple

Chung-Yi Chiu

I-Hua Chu

Angela Ciccia

Nancy Ciccollela

Angela Colantonio

Martha Combs

John Connolly

Kristin Jane Conover

Andrew Cook

Caitlin Cowan*

Christopher Cranston

John Cryan

Nickolas Dasher

Ed de St. Aubin

Charles Degeneffe

Victor DelBene*

Nicole Ditchman
Thomas Dixon

Ewa Domagała-Zyśk

Christina L. Duncan

Katherine M. Dunn*

Adva Eichengreen

Ipek Ensari

Rosa Esteve Zarazaga

Pamela Fitzpatrick

Theresa Floegel

Anjali J. Forber-Pratt

Robert T. Fraser

Katherine Parris Frey

Carli Friedman

Armando Fuentes*

Maryam Gholami

Christopher D. Graham

Jessica Graham

Kristin M. Graham

Juliet Haarbauer-Krupa

Nicholas Harland

Tyler Harris

Anthony Harrison

Tracie Harrison

James Henry

Adam Hirsh

Andrew Hoisington

Megan Hosey

Abbey Jean Hughes

Amy Jak

Alicia M. January

Brian Johnson

Erica K. Johnson

Salene M. W. Jones

Jennifer Jutte
Claire Z. Kalpakjian

Karen-Inge Karstoft

Paul King

Kristine Kingsley

Nikos Konstantinou

Caroline Lassen-Greene

Margaret Legarreta

Irene W. Leigh

Sheera F. Lerman

Allison Levine

Lena Lipskaya-Velikovsky

Eva Louvet

Junfei Lu

Emily M. Lund

Gloria Ma

Ellen Manegold*

Sarah L. Martindale

Shannon McCaslin

John McClure

Jed N. McGiffin

Swati Mehta

Laurie Miller

Ivan Molton

Kimberley R. Monden

Dennis Moore

Donna Morere

Helene Moriarty

Stephanie Moser

Emily Nalder

Sarah Nelson

Mara Nery-Hurwit

Barry P. Nierenberg

Deirdre O'Sullivan

Monika Parchomiuk

Carisa Perry-Parrish
Brian Nelson Phillips

Thomas Platz

Robyn Powell

Lisa Rapport

Jennifer Reesman

Dominique Reinwand

Diego Rivera

Ed Rohn

Henry Scharm*

Katharine Seagly

Ron T. Seel

Sophia Serpa*

Robert Brian Shaw

Richard L. Skolasky

Evan L. Smith

Amy Starosta

Lillian Flores Stevens

William Stiers

Douglas Strohmer

Kirk J. Stucky

John Andrew (Drew) Sturgeon

Alicia Swan

Gareth Terry

Salom Teshale*

Manu Thakral

Marta Tremolada

Brigid Waldron-Perrine

Amy Ward

Catherine Wilson

Jennifer Wilson

Steven Woods

Rana Yaghmaian

Mercedes Zapata

*Denotes Co-reviewer. 